\title{
Mutation-specific differences in arrhythmias and drug responses in CPVT patients: simultaneous patch clamp and video imaging of iPSC derived cardiomyocytes
}

\author{
R. P. Pölönen ${ }^{1}(1) \cdot$ H. Swan ${ }^{2} \cdot$ K. Aalto-Setälä ${ }^{1,3}(\mathbb{0}$
}

Received: 4 June 2019 / Accepted: 21 November 2019 / Published online: 30 November 2019

(c) The Author(s) 2019

\begin{abstract}
Catecholaminergic polymorphic ventricular tachycardia (CPVT) is an inherited cardiac disease characterized by arrhythmias under adrenergic stress. Mutations in the cardiac ryanodine receptor (RYR2) are the leading cause for CPVT. We characterized electrophysiological properties of CPVT patient-specific induced pluripotent stem cell-derived cardiomyocytes (hiPSC-CMs) carrying different mutations in RYR2 and evaluated effects of carvedilol and flecainide on action potential (AP) and contractile properties of hiPSC-CMs. iPSC-CMs were generated from skin biopsies of CPVT patients carrying exon 3 deletion (E3D) and L4115F mutation in RYR2. APs and contractile movement were recorded simultaneously from the same hiPSC-CMs. Differences in AP properties of ventricular like CMs were seen in CPVT and control CMs: APD90 of both E3D $(n=20)$ and L4115F ( $n=25)$ CPVT CMs was shorter than in control CMs $(n=15)$. E3D-CPVT CMs had shortest AP duration, lowest AP amplitude, upstroke velocity and more depolarized diastolic potential than controls. Adrenaline had positive and carvedilol and flecainide negative chronotropic effect in all hiPSC CMs. CPVT CMs had increased amount of delayed after depolarizations (DADs) and early after depolarizations (EADs) after adrenaline exposure. E3D CPVT CMs had the most DADs, EADs, and tachyarrhythmia. Discordant negatively coupled alternans was seen in L4115F CPVT CMs. Carvedilol cured almost all arrhythmias in L4115F CPVT CMs. Both drugs decreased contraction amplitude in all hiPSC CMs. E3D CPVT CMs have electrophysiological properties, which render them more prone to arrhythmias. iPSC-CMs provide a unique platform for disease modeling and drug screening for CPVT. Combining electrophysiological measurements, we can gain deeper insight into mechanisms of arrhythmias.
\end{abstract}

Keywords hiPSC $\cdot$ Cardiomyocyte $\cdot$ CPVT $\cdot$ Patch clamp $\cdot$ Contraction $\cdot$ Carvedilol $\cdot$ Flecainide

Electronic supplementary material The online version of this article (https://doi.org/10.1007/s11033-019-05201-y) contains supplementary material, which is available to authorized users.

R. P. Pölönen

risto-pekka.polonen@tuni.fi

H. Swan

heikki.swan@helsinki.fi

K. Aalto-Setälä

katriina.aalto-setala@tuni.fi

1 Faculty of Medicine and Health Technology, Tampere University, Arvo Ylpön katu 34, Arvo2 D441, 33520 Tampere, Finland

2 Helsinki University Hospital and Helsinki University, PO Box 340, 00029 Helsinki, Finland

3 Heart Center, Tampere University Hospital, Arvo Ylpön katu 34, Arvo2 D437, 33520 Tampere, Finland

\section{Introduction}

Human induced pluripotent stem cells (hiPSCs) can be generated from patients' somatic cells providing endless source for research in vitro [1]. The hiPSCs carry the same mutations as the patient who donated the primary cells and the differentiation of disease specific hiPSC-derived cardiomyocytes (CMs) allows disease modeling and drug development in human cells for personalized medicine.

Catecholaminergic polymorphic ventricular tachycardia (CPVT) is a severe inherited cardiac disease in structurally normal heart associated with risk of sudden cardiac death [2]. CPVT patients have arrhythmias during mental or physical stress. CPVT is caused by mutations mainly in the $R Y R 2$ gene, coding for cardiac ryanodine receptor (RYR), but also mutations in other genes have been observed [3]. Therapy includes drug treatment, usually with beta-blockers, and, in 
severe cases, implantable cardiac defibrillators or left cardiac sympathetic denervation. However, present therapeutic options do not provide complete protection against potentially lethal arrhythmias. A beta-blocker carvedilol and class Ic antiarrhythmic agent flecainide have been shown to have a direct effect on RYR and they, or their derivatives, are investigated as treatment options for CPVT [4-7].

$\mathrm{CM}$ contraction is driven by action potential (AP) induced release of intracellular $\mathrm{Ca}^{2+}$ from sarcoplasmic reticulum (SR) [8]. Inward current through sodium channels $\left(\mathrm{I}_{\mathrm{Na}}\right)$ depolarizes the $\mathrm{CM}$ cell membrane which triggers inward flow of $\mathrm{Ca}^{2+}$ through L-type calcium channels $\left(\mathrm{I}_{\mathrm{Ca}, \mathrm{L}}\right)$, which leads to activation of RYRs and calcium induced calcium release (CICR) from SR. $\mathrm{Ca}^{2+}$ binds to sarcomeres and allows the contractile motion. Cell membrane is repolarized by several potassium currents and $\mathrm{Ca}^{2+}$ is reloaded into SR via SR $\mathrm{Ca}^{2+}$ ATPase (SERCA) [9]. The membrane potential $\left(\mathrm{V}_{\mathrm{m}}\right)$ and intracellular $\mathrm{Ca}^{2+}$ concentration $\left[\mathrm{Ca}^{2+}\right]_{\mathrm{i}}$ are the most crucial elements in cardiac excitation contraction coupling (ECC) and arrhythmogenesis. The $\left[\mathrm{Ca}^{2+}\right]_{\mathrm{i}}$ influences the shape and duration of $\mathrm{AP}$ via $\mathrm{Ca}^{2+}$-sensitive ionic transporters, such as $\mathrm{I}_{\mathrm{Ca}}, \mathrm{Na}^{+}-\mathrm{Ca}^{2+}$ exchanger (NCX) and $\mathrm{Ca}^{2+}$-activated nonselective cation channels, thereby modifying the electrophysiological properties for example refractoriness and membrane depolarization rate [9]. Conversely, membrane potential can influence the $\mathrm{Ca}^{2+}$ spark and waves [10].

A few studies have been focused on simultaneous recording of $\mathrm{V}_{\mathrm{m}}$, calcium transients or contractile function from the same hiPSC CMs [11-19]. It is important to understand the highly inter-related nature between these key elements in ECC. The ability to combine ionic and biomechanic analysis can offer valuable details considering disease mechanisms, drug effects and arrhythmogenesis. In our previous studies, we have measured intracellular calcium, action potentials and field potentials from CPVT hiPSC CMs [20-23]. In this study, we combined current clamp recording with video imaging. We characterized electrophysiological and contractile properties of CPVT patient-specific hiPSC CMs carrying different mutations in RYR2. In addition, we evaluated effects of carvedilol and flecainide, agents that are shown to affect RYR2, on APs and contractility of hiPSC-CMs.

\section{Materials and methods}

\section{Patient-specific human iPSC lines}

In this study, two CPVT hiPSC lines were used carrying following RYR2 mutations: one with exon 3 deletion c.168301_c. 273+722del1128 (E3D) and one with point mutation p.L4115F (c.12343C > T) (L4115F). Mutation nomenclature was based on RYR2 reference sequence NM_001035.2.
Wild type hiPSC line from one healthy individual was used as a control cell line. Several clones were established from the individuals but one per each was used in this study for characterization. The ethical committee of Pirkanmaa Hospital District approved collection of biopsies for generating patient-specific iPSC lines and written informed consent was obtained from all the donors (Aalto-Setälä R08070). Human iPSC-lines were established by sendai viral (CytoTune ${ }^{\circledR}$ iPS reprogramming kit, Thermo Fisher Scientific, Waltham, MA, USA) or retroviral transfection of OCT3/4, SOX2, KLF4 and c-MYC [1]. Characterization of hiPSC lines has been described previously [22].

\section{Cardiac differentiation and dissociation}

Differentiation into CMs was carried out by co-culturing hiPSCs with murine visceral endoderm-like (END-2) cells (prof. Mummery, Humbrecht Institute, Utrecht, The Netherlands) (supplementary data) [24]. Human iPSCs formed spontaneously beating clusters after 15 days of co-culturing. Beating clusters were cut and isolated with scalpel and dissociated with collagenase A (Roche Diagnostics) [24]. Not all beating hiPSC CM clusters dissociate into single cells, therefore resulting into suspension of mixed single cell CMs and small $\mathrm{CM}$ aggregates. These $\mathrm{CMs}$ were plated on glass coverslips coated with $0.1 \%$ gelatin.

\section{Chemicals}

All the drugs and chemicals for the experiments were purchased from the Sigma-Aldrich unless otherwise specified. Potassium methanesulfonate (KMeSO4) was ordered from MP biomedicals (USA). Stock solutions for extracellular solution were prepared in $1 \mathrm{M}$ concentration and refrigerated until use. Extracellular solution was made freshly by diluting stock solution (in mM): $143 \mathrm{NaCl}, 4.8 \mathrm{KCl}, 1.8 \mathrm{CaCl}_{2}, 1.2$ $\mathrm{MgCl}_{2}, 5$ glucose and 10 HEPES (pH adjusted to 7.4 with $\mathrm{NaOH}$ ). Intracellular solution was made and refrigerated (in $\mathrm{mM}$ ): $132 \mathrm{KMeSO} 4,4$ EGTA, $20 \mathrm{KCl}, 1 \mathrm{MgCl}_{2}$ and 1 $\mathrm{CaCl}_{2}$ (pH adjusted to 7.2 with $\mathrm{KOH}$ ). Adrenaline, carvedilol and flecainide were dissolved in Milli- $\mathrm{Q}^{\mathrm{TM}}$ water and stored frozen until diluted to the final concentration $(1 \mu \mathrm{M}$, $0.25 \mu \mathrm{M}$ and $1,5 \mu \mathrm{M}$ respectively) in extracellular solution at the day of the experiments. Amphotericin-B was dissolved in DMSO and diluted to intracellular solution to make a final concentration of $0.24 \mathrm{mg} / \mathrm{mL}$. One drug (either carvedilol or flecainide) was used per each cardiomyocyte. Same cardiomyocytes were not exposed to both drugs.

\section{Simultaneous patch clamp and video imaging}

Experiments were conducted on $30+$ days old (from start of differentiation) hiPSC CMs which were plated on cover 
slips no more than 7 days before the experiments. Extracellular solution was heated to $36 \pm 1{ }^{\circ} \mathrm{C}$ with an inline heater SH-27B controlled with a TC-324B controller unit (Warner instruments Inc., CT, USA). The perfusion was controlled by a gravity driven VC38 application system (ALA Scientific Instruments Inc., NY, USA). Patch pipettes (Harvard Apparatus Ltd., Holliston, MA, USA) were freshly prepared using PC-10 micropipette puller and flame polished with MF-830 microforge (both from Narishige Int., Tokyo, Japan). The patch electrodes had tip resistance of 1.8-2.2 $\mathrm{M} \Omega$ with intracellular solution. AP recordings and video imaging were performed simultaneously. For patch clamp experiment, perforated patch using Amphotericin B was performed to record AP in Axon Series 200B patch-clamp amplifier connected to Digidata 1440a AD/DA converter driven by pCLAMP 10.2 software (all from Molecular devices LLC). APs were recorded in the gap-free mode in the current-clamp from the spontaneously beating hiPSC-CMs. Current-clamp recordings were digitally sampled at $20 \mathrm{kHz}$ and filtered at $2 \mathrm{kHz}$ using a low pass Bessel filter on the recording amplifier. Videos were captured with ANDOR iXon 885 EM-CCD camera (Andor Technology, Belfast, Northern Ireland) and a TH4-200 light source (Olympus, Tokyo, Japan). Video imaging was performed using an inverted Olympus IX70 microscope using LCAch N PhC 0.40NA $20 \times$ air objective (Olympus) and recorded with LiveAcquisition software (TILL Photonics, Munich, Germany). The videos were recorded as 30 s-long sweeps with 49-105FPS before and after drug administration. Without binning of the camera and relatively large active pixel size, the videos were not the highest FPS possible. After establishing the seal, minimum of 2 min was allowed for the cardiomyocytes to stabilize before recording the baseline condition. The pCLAMP software was configured to perform the synchronization between patch clamp and video imaging system by receiving the synchronization pulse (SP) from the camera: SPs were recorded alongside with APs, thus when SP stopped, the video imaging was stopped.

\section{Action potential and contraction analysis}

Action potentials were analyzed with Origin ${ }^{\mathrm{TM}} 9.1$ (OriginLab Corp., Northampton, USA) to extract the action potential parameters. The hiPSC-CMs were categorized as ventricular-like and atrial-like CMs when they showed APD90/ APD50 $<1.30$ and APA $>80 \mathrm{mV}$, and APD90/APD50 $>1.35$, respectively. The different arrhythmias shown in the Fig. 3 were analyzed manually. DADs were defined as brief $>5 \mathrm{mV}$ depolarizations in the root or close proximity of the AP at the terminal repolarization (Fig. 3e, g, h). We have earlier characterized the average DAD amplitude as $5-15 \%$ of the preceding AP [18]. EADs were identified as extra AP or consecutive AP burst after the initial AP (Fig. 3g, h). Alternans was identified as constant alternating APD (long-short-long) for ten beats or longer (Fig. 3i). Tachyarrhythmia was identified as a transient change in BPM (Fig. 3j). Frequency of DADs and EADs was calculated by dividing the number of arrhythmias with the recorded time in each condition. The contraction properties were analyzed with ImageJ (NIH) plugin Musclemotion [25] with its built-in noise reduction and automatic reference frame detection settings. Cell size was also measured with ImageJ. The analysis was conducted only after minimum of $2 \mathrm{~min}$ to let the cell and Aps stabilize.

\section{Statistical analysis}

Statistical analysis was carried out with SPSS software version 23 (SPSS, Chicago, IL, USA). Comparison within cell lines (before and after drug administration) were performed with non-parametric Wilcoxon and between cell lines (cell line to cell line comparison) with non-parametric Mann-Whitney U tests with Dunn-Bonferroni post hoc. $\mathrm{p}<0.05$ was considered statistically significant. Correlation of different parameters was done with Pearson correlation test.

\section{Results}

\section{Differences between $\mathrm{CM}$ aggregates and single cells}

There were no significant differences between AP or contraction parameters between CM aggregates and single cells (Tables S1 and S2). In this dataset, 68\% were CM aggregates and $32 \%$ were single CMs. An aggregate consisted usually of two or few cells connected to each other (Fig. 3a-d, i, j). Area of CM aggregate was larger than the average cell area of single CM, approximately $8280 \mu \mathrm{m}^{2}$ and $5370 \mu \mathrm{m}^{2}$ respectively (Fig. S1). Single CMs were more spread out than the ones inside an aggregate (Fig. 3e-h). In addition, there was no significant correlation between cell area and any AP or contraction parameter. Therefore, results from aggregates and single cells were pooled.

\section{Action potential parameters and compound effects}

CMs were recorded in current clamp and AP parameters were extracted (Fig. 1). Most of the CMs were ventricular like with APD90/50 ratio lower than 1.30. Properties of atrial like hiPSC CMs are shown in Table S3. Nodal like CMs were very few in number (data not shown). Only ventricular like CMs were studied further with adrenaline and then with either carvedilol or flecainide along with adrenaline. Representable traces from compound effects are in Fig. 3a-f. 

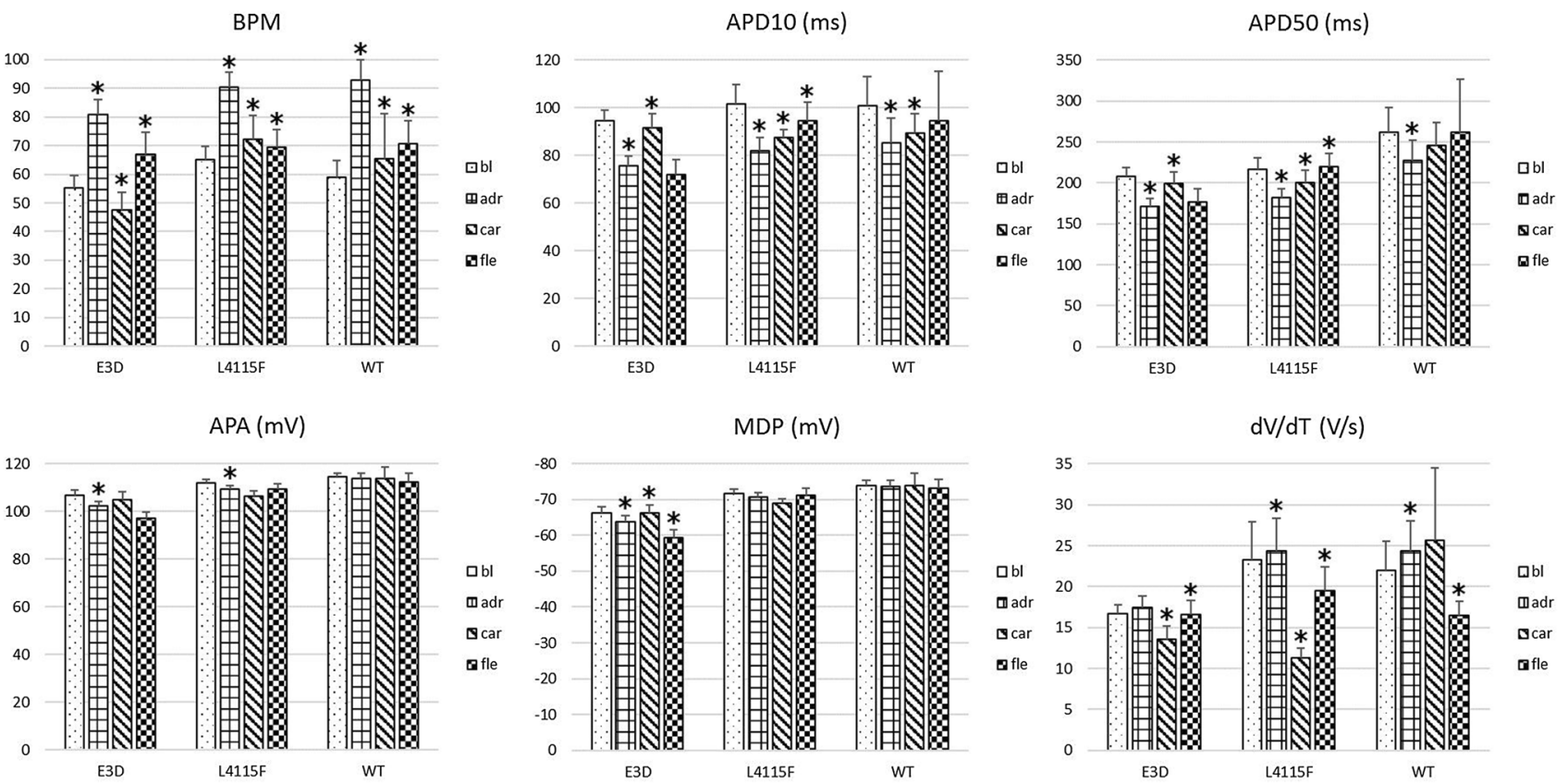

Fig. 1 Action potential parameters and compound effects on ventricular like control and CPVT hiPSC CMs. $\mathrm{Bl}=$ baseline, $\mathrm{adr}=$ adrenaline, $\mathrm{car}=$ carvedilol, fle $=$ flecainide. Number of cells recorded: E3D) $\mathrm{bl}=20, \mathrm{adr}=20, \mathrm{car}=13, \mathrm{fle}=7 ; \mathrm{L} 4115 \mathrm{~F}) \mathrm{bl}=25, \mathrm{adr}=25$, $\mathrm{car}=6$, fle $=19$; WT) $b l=15, \operatorname{adr}=15, \mathrm{car}=5$, fle $=10$. BPM $=$ beats per minute, $\mathrm{APD}=$ action potential duration at given $\%$ of repolarization,

At baseline, hiPSC CMs differed from one to another: L4115F-CPVT CMs had the highest beating rate, E3DCPVT CMs had the lowest APA and MDP $(\mathrm{p}<0.05)$. Overall, APD90 and APA was lower and MDP more depolarized in CPVT compared to control CMs. Adrenaline had positive chronotropic effect on all hiPSC CMs: Beating rate was increased and APD10, 50 and 90 were decreased $(\mathrm{p}<0.05)$. In addition, adrenaline decreased APA and depolarized MDP the most in E3D-CPVT CMs ( $<<0.05)$. Furthermore, adrenaline increased upstroke velocity ( $\mathrm{dVdT}$ ) and triangularity (APD ratio) in $\mathrm{L} 4115 \mathrm{~F}$ and WT CMs $(\mathrm{p}<0.05)$ but not in E3D CPVT CMs. Carvedilol and flecainide both had negative chronotropic effect on all hiPSC CMs: decreased beating rate and increased APD90 $(\mathrm{p}<0.05)$. In addition, both drugs decreased upstroke velocity and increased triangularity in CPVT CMs $(\mathrm{p}<0.05)$. Carvedilol decreased beating rate the least but upstroke velocity the most in L4115F-CPVT CMs. Carvedilol had the least effect on APDs in control CMs. Flecainide decreased upstroke velocity the least but increased APD50 and 90 the most in L4115F CPVT CMs.

\section{Contraction parameters and drug responses}

Contraction properties of hiPSC CMs were studied with video imaging simultaneously along current clamp recording. Not all the videos could be analyzed in such a
$\mathrm{APA}=$ action potential amplitude, $\mathrm{MDP}=$ maximum diastolic potential, $\mathrm{dVdT}=$ upstroke velocity, APD90/50 = triangularity. ${ }^{*}$ Indicates statistical significance $(\mathrm{p}<0.05)$. Statistical comparisons were done between adr versus bl and car or fle versus adr. Error bars represent standard error of mean

precision because of low signal to noise ratio, therefore the $\mathrm{n}$ amount in contraction parameters in Fig. 2 is less than in AP parameters in Fig. 1. In addition, the comparison of contraction amplitude (CTA) was not plausible between recordings because of variation in the lighting conditions affected by the liquid flow during each recording. The preheated perfusate created a turbulent flow and small bubbles in the recording chamber resulting in diffraction of light and different lighting and baseline conditions for the intensity based peak detection. Therefore, only the compound effects as the change of CTA can be compared within one recorded cell. At baseline, CPVT CMs had shorter contraction duration at $90 \%$ of relaxation (CTD90) and relaxation time (Fig. 2). Adrenaline decreased CTDs, time to peak and relaxation time more in controls than CPVT CMs. Flecainide prolonged CTDs and relaxation time the most in control CMs. Both drugs decreased contraction amplitude the most in E3D CPVT CMs. Interestingly, flecainide decreased the relaxation time and CTDs only in E3D CPVT CMs. In addition, flecainide decreased the CTA the most in E3D CPVT CMs. Carvedilol decreased the contraction amplitude more than flecainide in L4115F CPVT and control CMs but not in E3D CPVT CMs. Furthermore, carvedilol decreased CTDs the most in control CMs. 

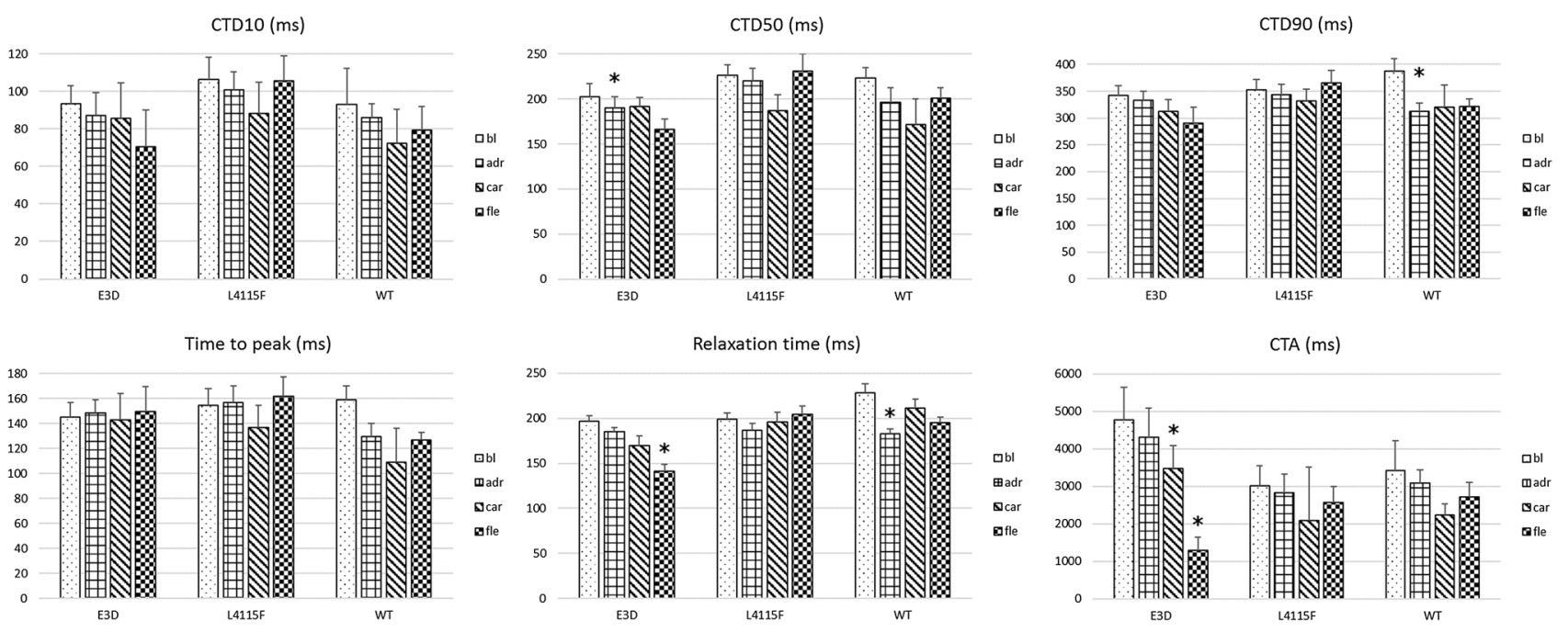

Fig. 2 Contraction parameters and compound effects on ventricular like control and CPVT hiPSC CMs. $\mathrm{Bl}=$ baseline, $\mathrm{adr}=$ adrenaline, car $=$ carvedilol, fle $=$ flecainide. Number of cells recorded: E3D) $\mathrm{bl}=18, \mathrm{adr}=18, \mathrm{car}=10, \mathrm{fle}=6 ; \mathrm{L} 4115 \mathrm{~F}) \mathrm{bl}=23, \mathrm{adr}=23, \mathrm{car}=6$, fle $=16$; WT) $b l=11, \operatorname{adr}=11, \quad$ ar $=6$, fle $=5$. CTD $=$ contraction duration at given percentage of relaxation, $\mathrm{CTA}=$ contraction ampli-

\section{Correlation between AP and contraction}

There was significant $(\mathrm{p}<0.01)$ positive correlation between all respective APD and CTD parameters (Fig. S2). Figure 3 merged sections show the interrelation between $\mathrm{V}_{\mathrm{m}}$ and contraction. Contraction follows $\mathrm{V}_{\mathrm{m}}$ in various arrhythmic conditions. Weak but significant negative correlation between BPM and time to peak was found $(\mathrm{p}<0.01)$ at baseline and adrenaline: time to peak decreases when beating rate increases. In addition, APD ratio and relaxation time also had weak and significant negative correlations $(\mathrm{p}<0.01)$ at baseline and adrenaline: the smaller the ratio, the longer the relaxation time. After drug administration, the correlations were not as strong or significant anymore. Carvedilol and flecainide therefore had an impact on coupling of membrane $\mathrm{V}_{\mathrm{m}}$ and contraction.

\section{Arrhythmias seen in patch clamp recording reflected on contraction}

None of the control CMs had arrhythmias at adrenaline whereas all the CPVT CMs were arrhythmic. Overall, hiPSC CMs had more DADs than EADs (Fig. 4). Representative traces of different arrhythmias are presented in Fig. 3. E3D CPVT CMs had the most arrhythmias at all conditions. Furthermore, CPVT CMs had increased amount of DADs and EADs after adrenaline exposure, however this result was not statistically significant $(\mathrm{p}>0.05)$. DADs are seen as a swift depolarization immediately after repolarization (Fig. 3g). These minor changes in $\mathrm{V}_{\mathrm{m}}$ are not reflected in contraction tude (normalized to control). The comparison of CTA is not plausible between different cells, only the proportional change of CTA can be compared. *Indicates statistical significance $(\mathrm{p}<0.05)$. Statistical comparisons were done between adr versus bl and car or fle versus adr. Error bars represent standard error of mean

profile. Phase 3 and burst EADs are present in both Vm and contraction (Fig. 3g, h). The extra beats resulting from EADs have decreased contraction amplitude. Both drugs were able to decrease the amount of DADs in CPVT CMs whereas flecainide slightly increased EADs in E3D CPVT CMs (Fig. 4). Carvedilol abolished almost all arrhythmias in L4115F CPVT CMs while flecainide decreased DADs 67\% and EADs $49 \%$ (Fig. 3c-f). In addition, flecainide decreased DADs $60 \%$ and carvedilol $51 \%$ in E3D CPVT CMs. APD alternans was seen in 2/25 (8\%) L4115F CPVT CMs after adrenaline as alternating APD in $\mathrm{V}_{\mathrm{m}}$ and amplitude in contraction (Fig. 3I). Long AP results in lower amplitude and vice versa. Flecainide abolished both of the alternans cases. Tachyarrhythmia were seen in 4/20 (20\%) E3D CPVT CMs and 1/25 (4\%) L4115F CPVT CMs as a rapid major change in beat rate and depolarized MDP continuing for over several seconds (Fig. 3j). Flecainide abolished one of the tachyarrhythmias, whereas it caused two CMs undergo tachyarrhythmia. Carvedilol abolished three of the four tachyarrhythmia cases.

\section{Discussion}

Following the discovery of linking RYR2 mutations to CPVT, studies utilizing patch clamp in RYR2-R4496C mutant mouse model have been published [2, 26-28]. Increased open probability of mutated RYR 2 leading to spontaneous calcium release and lowered threshold for triggered activity has been identified as a primary cause 
AP

A

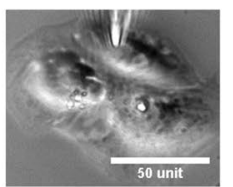

B

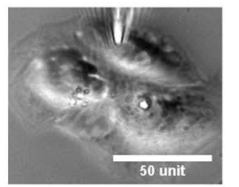

C

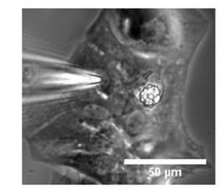

D

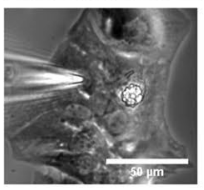

E

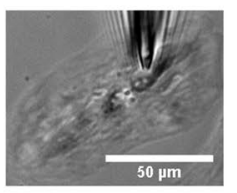

F

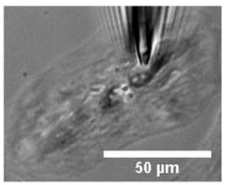

G

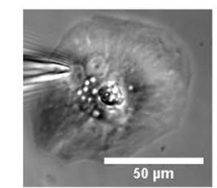

$\mathrm{H}$

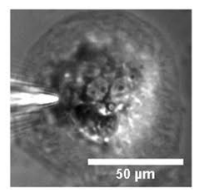

I

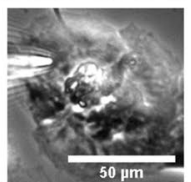

]

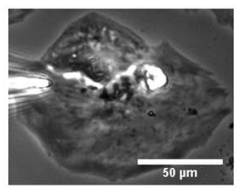

Contraction
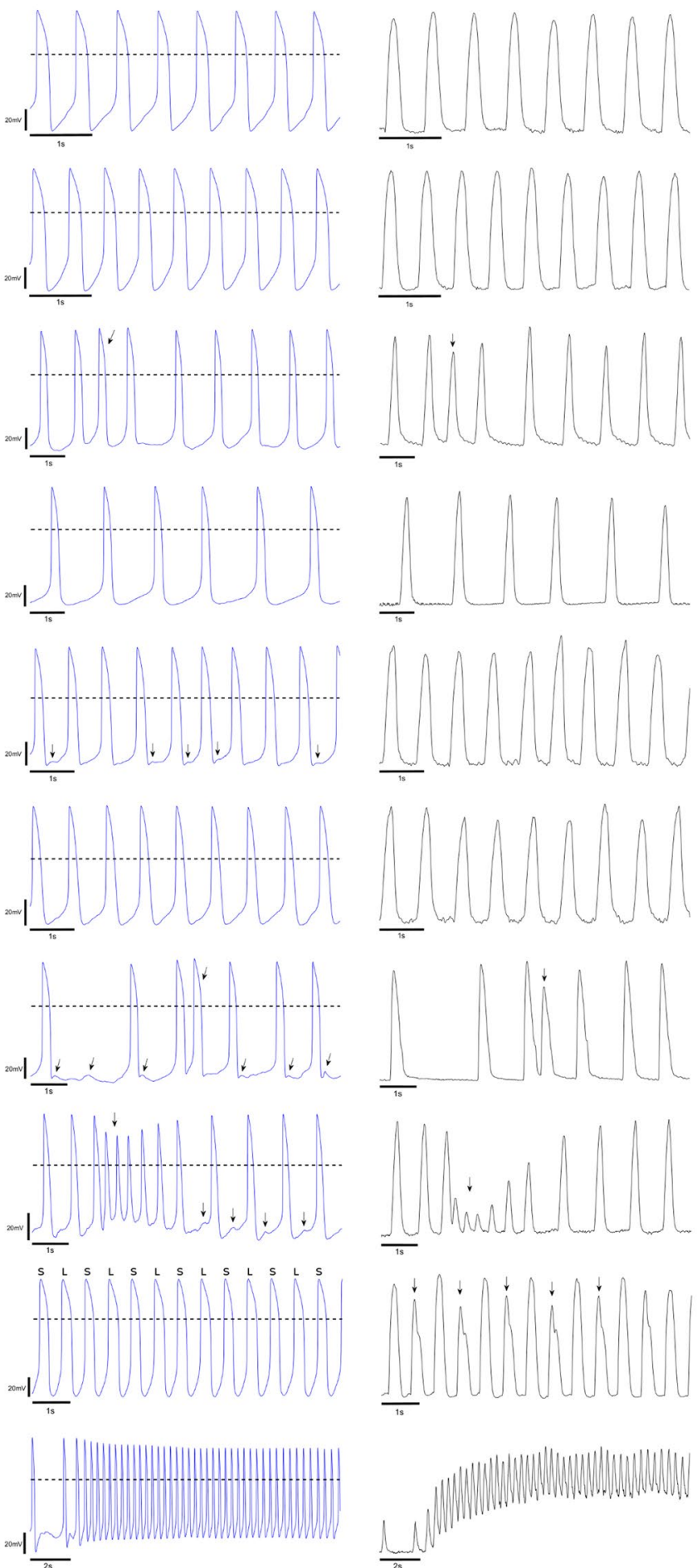

Merge
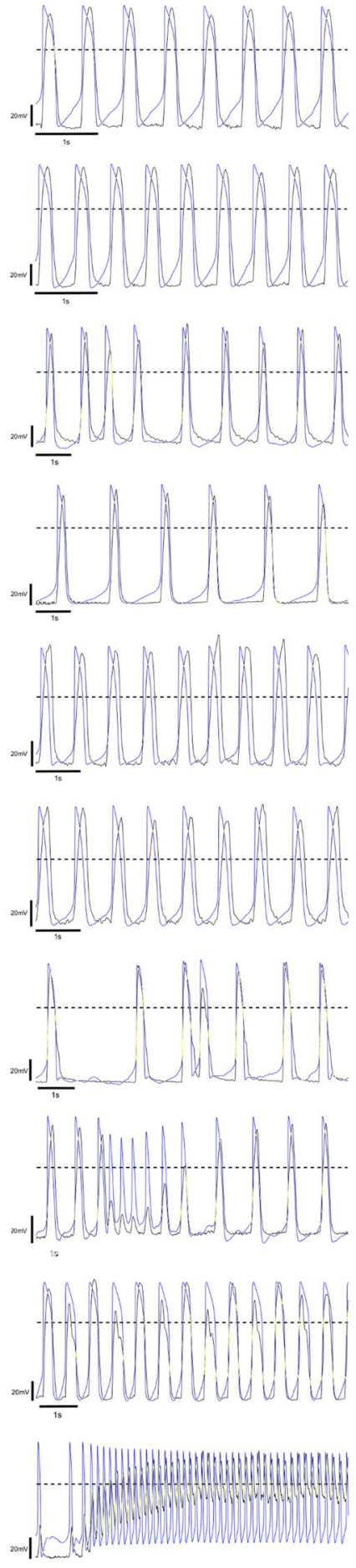

Fig. 3 Representative traces of drug responses and arrhythmias in hiPSC CMs. Action potential and contraction measured simultaneously from the same cell. a Normal baseline trace of WT control, b normal adrenaline trace from the same cell as in $(\mathbf{a}, \mathbf{c})$ adrenaline trace from CPVT showing extra beat (arrow), $\mathbf{d}$ carvedilol trace from the same cell as in (c, e) adrenaline trace from CPVT showing DADs (arrows), $\mathbf{f}$ flecainide trace from the same cell as in (e, g) DADs and phase 3 EADs (arrows) from CPVT CM, h EAD burst and DADs (arrows) from CPVT CM, i alternans from CPVT CM where long AP (L) results in lower contraction amplitude (arrow) and short AP (S) in higher, $\mathbf{j}$ tachyarrhythmia from CPVT CM. Dashed lines at $0 \mathrm{mV}$ 
for DADs and arrhythmia. Patch clamp recordings showing DADs under isoproterenol or other adrenergic stimulus have confirmed this. However, electrophysiological properties of mouse and human CMs are different, e.g. beating rate and presence of repolarizing ionic currents [29]. Therefore, hiPSC derived CMs have been introduced, and electrophysiology studies using CPVT patient derived hiPSC CMs have been published, and the disease phenotype has been confirmed in human-based cell model in vitro [23, 30-32].

In this study, we characterized electrophysiological properties of E3D and L4115F CPVT hiPSC-CMs and control $\mathrm{CMs}$ at baseline and after adrenaline provocation and evaluated antiarrhythmic effects of carvedilol and flecainide. CPVT CMs had shorter APD90 and more depolarized MDP compared to controls at baseline. The more depolarized MDP led to lower APA in CPVT CMs. In addition, the shorter APD90 probably was caused by the sarcolemmal calcium transient affected via the leaky RYR2 mediated higher intracellular calcium concentration. Furthermore, MDP change affects the availability of ion channels at the cardiomyocyte membrane laying effect on the AP profile. Adrenaline had positive and carvedilol and flecainide negative chronotropic effect in all hiPSC CMs. CPVT CMs had increased number of DADs and EADs after adrenaline exposure. Adrenaline provocation increases the SR calcium load and results to calcium leak via the mutated RYR2s. The results suggest that the depolarized MDP plays a role in the generation of arrhythmias in E3D CPVT CMs. Alternans was seen in L4115F CPVT CMs. Carvedilol and flecainide decreased the number of DADs but flecainide increased EADs in E3D CPVT and control CMs. In addition, both drugs decreased contraction amplitude in all hiPSC CMs.

\section{CPVT CMs had more DADs than EADs}

In this study, CPVT hiPSC CMs were more arrhythmic than control CMs (Fig. 4). In addition, there were more DADs than EADs in CPVT CMs. Carvedilol was more efficient abolishing EADs than flecainide (Fig. 4b). Carvedilol acts by blocking beta-adrenergic receptors, inhibiting phosphorylation of calcium handling proteins (RYR, SERCA, $\mathrm{I}_{\mathrm{Ca}, \mathrm{L}}$ ) and altering calcium handling. Flecainide acts by blocking sodium channels leading to reduced excitability, but also $\mathrm{I}_{\mathrm{Kr}}$ leading to risk for arrhythmia. In addition, both drugs have shown to reduce RYR2 calcium leak associated with DADs [4-7]. In this study, both drugs decreased the number of DADs in CPVT CMs (Fig. 4).

Novak et al. reported oscillatory prepotentials (OPPs), distinct from DADs, described as voltage oscillations during late diastolic depolarization [32, 33]. This irregularity in the membrane potential is very similar to what have been earlier described as DADs [23, 27, 28, 30, 31]. In our current study, flecainide abolished majority of DADs but not all of them. In Fig. 3e, g, h, two kind of DADs appear: right after repolarization in the root of AP (Fig. 3e) and later in the diastolic depolarization (Fig. 3h). The latter hold a remarkable resemblance to OPPs. Flecainide cured all of the first mentioned DADs, but only a portion of the latter. This could indicate distinctive ways these two types of DADs are produced. However, the APD prolongation caused by flecainide may mask the first type of DADs. Furthermore, some CMs exhibited long irregular periodical beating patterns (Fig. S3). There is similar voltage oscillation during OPPs and the gaps between these periods. This might be due to aberrant CM automaticity and different from the mechanism of DADs. Cells, which were beating inconsistently in this manner, were excluded from the further analysis.

\section{E3D CPVT CMs: depolarized MDP and high DAD frequency}

In this study, E3D CPVT CMs had the most DADs and EADs. The more positive, depolarized MDP seems to be favoring arrhythmic events by setting lower threshold for triggered activity. SR calcium leak through RYR can lead to small changes in membrane voltage leading to activation of $\mathrm{I}_{\mathrm{Ca}, \mathrm{L}}$ or influx through NCX and eventually CICR, therefore causing premature action potential triggering and extra beats. Consequently, APA and upstroke velocity (dVdT) were also smaller in these cells.

In earlier studies, the exon 3 deletion in RYR2 leads to a severe clinical phenotype of CPVT [34-36]. At the protein level, exon 3 deletion causes a flexible rearrangement in the cytosolic part of the RYR2 affecting domain-domain interactions inside the protein [35, 37]. Yet another study found the exon 3 deletion of RYR2 to result in the abnormal termination of calcium release through the channel [38]. These aforementioned studies support our earlier study, where E3D CPVT patient was shown to have more polymorphic ventricular contractions at rest compared to the L4115F CPVT patient [20]. Similarly, in this study there were more arrhythmias in E3D CPVT hiPSC CMs at baseline. In addition, similarly to this study, in that same earlier study, the beating rate of the E3D CPVT CMs was lower than the L4115F CPVT CMs [20]. Furthermore, in another of our previous studies, the expression of the RYR2 protein was confirmed and there was no difference between RYR2-P2328S CPVT and control CMs [23]. In that same study, fractional SR calcium release was also found greater in those CPVT CMs compared to controls.

In our earlier calcium imaging study of the same E3D CPVT CMs, there were the most oscillating calcium transients, which were several seconds in duration, in these CMs [22]. That is probably why there is such a difference when comparing the calcium transient duration in 
(A) DADs / $\min$

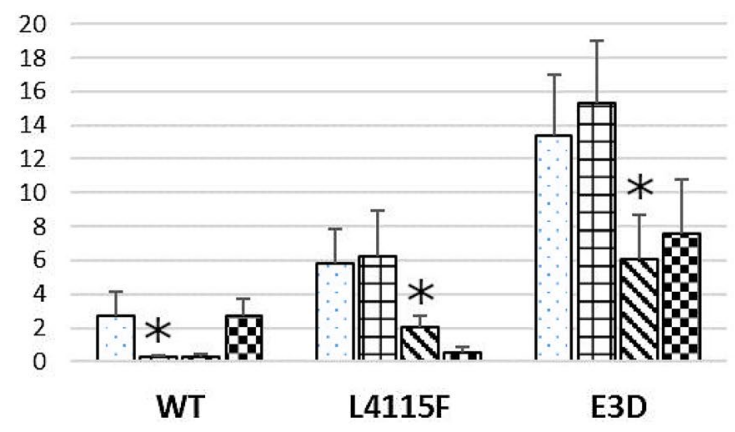

$\square$ bl $\square$ adr $\mathbf{\Delta f l e} \mathbf{Q}$ car
Fig. 4 Incidence of arrhythmias in hiPSC CMs. Bl=baseline, $\mathrm{adr}=$ adrenaline, $\mathrm{car}=$ carvedilol, fle $=$ flecainide. Number of cells recorded: $\mathrm{E} 3 \mathrm{D}) \mathrm{bl}=20, \mathrm{adr}=20, \mathrm{car}=13, \mathrm{fle}=7$; $\mathrm{L} 4115 \mathrm{~F}) \mathrm{bl}=25$, $\operatorname{adr}=25, \operatorname{car}=6, \mathrm{fle}=19 ; \mathrm{WT}) \mathrm{bl}=15, \mathrm{adr}=15, \mathrm{car}=5, \mathrm{fle}=10$.

that study to APDs in this study. In addition, the beating rates of the CMs were different. Since the repolarization of AP is much more rapid than calcium decay, similar oscillation patterns were not detected in membrane voltage in this study. In addition, it could be that the CMs exhibiting periodical beating were the ones captured with the long calcium oscillations in the previous study, and in this study, they were not included in the ventricular like dataset. In addition, calcium imaging does not allow distinguishing between CM subtypes. Furthermore, the previous study showed that E3D CPVT CMs had lower calcium transient amplitude, which could be an indication of elevated diastolic calcium [22]. We returned to that calcium imaging dataset and analyzed the baseline fluorescence intensity values at diastole and indeed, in CPVT cells it was higher compared to the controls and the highest in E3D CPVT CMs $(p<0.001)$ (Fig. S4). These findings suggest that the RYR2 exon 3 deletion results in elevated diastolic calcium, which contributes to the depolarized diastolic potential therefore facilitating arrhythmic behavior in these cells.

\section{Alternans}

Similar to our previous study, L4115F CPVT CMs had alternans during adrenergic stress [22]. In this study, two alternans cases were recorded, both in L4115F CPVT CMs. Both of these were discordant, negatively coupled and electromechanically out of phase, in which the long AP results in low and short AP in high contraction amplitude (Fig. 3i). It has been shown with in silico modeling that in these cases the driving force of the alternans is unstable intracellular
(B) EADs / min

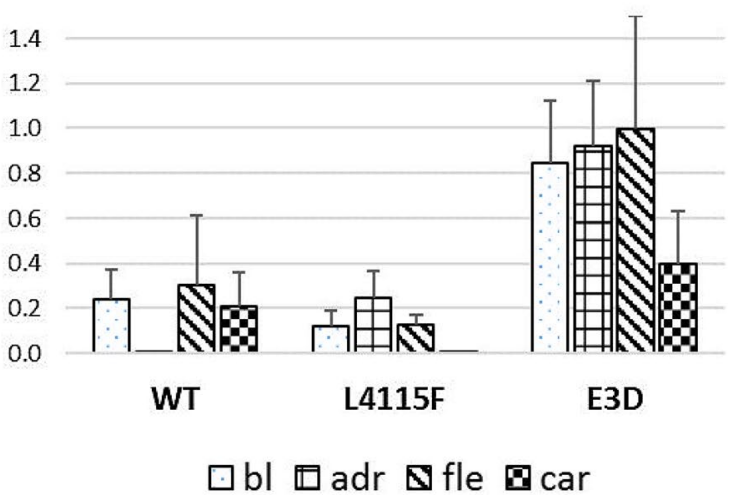

EADs include phase 3 and burst EADs. *Indicates statistical significance $(\mathrm{p}<0.05)$. Statistical comparisons were done between adr versus bl and car or fle versus adr. Error bars represent standard error of mean

calcium [39]. In addition, the RYR2-L4115F point mutation is located in the central domain of the protein leading to gain of function of the channel and could therefore facilitate the formation of alternans via imbalanced calcium cycling [40]. In this study, we did not conduct calcium imaging but it is known from the previous studies that calcium and contraction go hand in hand [15]. Membrane voltage, calcium and contraction are tightly coupled in cardiac ECC. In addition to causing APD alternans, we showed that calcium alternans can cause very small scale MDP alternans leading to more negative MDP after a long AP and a bit more positive after a short one (Fig. S5). MDP changes between consecutive beats were only approximately $0.5 \mathrm{mV}$. This indicates that higher calcium content during longer calcium transient, associated with the short AP, contributes to the repolarization to a more positive MDP.

\section{Contraction of hiPSC CMs}

Contraction parameters were acquired by analyzing videos of beating CMs. Sampling interval at lower video frame rates can affect the accuracy of the analysis of rise time of the contraction peaks. We used larger active camera chip without binning to capture the whole cell area of the CMs and CM aggregates for the analyses. In addition, contraction parameters had relatively high deviation (Fig. 2). Consequently, there was a lack of statistical significance in these results. Figure 3 shows how different arrhythmias were recapitulated in contraction profile. EAD triggered extra beats were characterized with lower contraction amplitude (Fig. 3g, h). Discordant negatively coupled alternans led to electromechanically out of phase beating pattern (Fig. 3i). Tachyarrhythmia 
elevated the baseline of contraction drastically but did not reduce contraction amplitude. Carvedilol decreased CTA, as a beta-blocker should, by reducing SR calcium load via inhibition of the adrenergic pathway. Carvedilol decreased the CTA more than flecainide in L4115F CPVT and control CMs, but not in E3D CPVT. This might be an indication of flecainide's improved block of RYR2 carrying the deletion. In E3D CPVT CMs, flecainide might be compensating the absence of beta-blocker ability by increased RYR2 block therefore resulting in as strong CTA decrease as carvedilol. Flecainide decreased CTDs most in E3D CPVT CMs. At more positive diastolic potential, APs are driven more by calcium than at more negative diastolic potentials because $\mathrm{I}_{\mathrm{Ca}, \mathrm{L}}$ open at more positive membrane potential than sodium channels. Interestingly, E3D CPVT CMs had higher contraction amplitude at baseline. Flecainide blocking both voltage gated sodium channels and RYR2 results in decreased CTDs and more decreased contraction amplitude compared to CMs with more repolarized MDP. Carvedilol, however, affects the SR calcium content by inhibition of adrenergic pathway and RYR2 block. The different pathways of action of these two drugs might explain the differences.

\section{Future perspectives}

Detailed investigation of electrophysiological properties of hiPSC CMs gives valuable information about arrhythmic conditions and their triggers, even though they do not completely recapitulate the phenotype of adult human CMs. However, they can serve several different purposes, for example drug screening and disease modeling. In addition, more detailed and fine-tuned in silico models can be developed to simulate human cardiac/cardiomyocyte physiology [41]. These cell models and approaches can be utilized by pharmacological industry to fulfil the need of more cost efficient and human-based drug development already in preclinical phases. Human iPSC derived CMs can offer physiologically more authentic model to study cardiotoxic effects in vitro, decrease the amount of animal studies and cut costs in the drug development process. Combining in vitro and in silico modeling will hopefully accelerate the cardiovascular research in the future.

\section{Conclusions}

In this study, simultaneous patch clamp and video imaging was conducted with CPVT patient derived hiPSC CMs to study effect of carvedilol and flecainide on AP and contractile properties. Differences in baseline properties and drug effects were found. CPVT CMs were more arrhythmic than controls. In addition, there were more DADs than
EADs in the hiPSC CMs. The E3D CPVT CMs had the most arrhythmias and electrophysiological properties distinct from others favoring arrhythmic behaviour. Different types of arrhythmias in membrane voltage were reflected in the contraction profile of the CMs. In this study, there were still substantial amount of DADs present after drug administration, indicating these treatments provide only partial protection from arrhythmias. Therefore, it is important to look for additional drug therapies for CPVT. Patient specific hiPSC CMs recapitulate disease hallmarks and drug responses therefore providing a powerful tool to study CPVT.

\section{Limitations}

In this study, only one clone per hiPSC line from a healthy donor was used to create control CMs. APD ratio was used to distinguish CM subtypes. However, controversy for the determination of the CM subtypes exists [42]. The immaturity of the hiPSC derived CMs, their calcium handling and electrophysiology; especially $\mathrm{I}_{\mathrm{Kf}}$ and $\mathrm{I}_{\mathrm{K} 1}$ is still a relevant problem [43]. Therefore, these results may not be translatable to patients. However, in our earlier studies, our hiPSC derived CMs have been characterized by various techniques showing their electrophysiological properties $[18,22,23,44]$.

Acknowledgements The authors thank Henna Lappi and Markus Haponen for assistance in cell culture and Chandra Prajapati and Kim Larsson for technical assistance in patch clamp. RPP carried out the experiments and analyzed the data and wrote the manuscript, HS collected the biopsies, KAS worked the manuscript and designed the study. All authors read and approved the final manuscript. This work was financially supported by Academy of Finland, the Finnish Cultural Foundation, the Finnish Foundation for Cardiovascular Research, Paavo Nurmi foundation and the Pirkanmaa Hospital district. The authors acknowledge Tampere Facility of Electrophysiological Measurements for their service. Abstract of this manuscript has been presented in Europhysiology 2018 conference, London UK, organized by Scandinavian Physiological Society, The Physiological Society and The Deutsche Society of Physiology and The Annual meeting of Finnish Developmental Biologists 2018 at Murikka, Finland. The manuscript has been used in the $\mathrm{PhD}$ thesis "Modeling Arrhythmias and Drug Responses in CPVT Patient Specific hiPSC Derived Cardiomyocytes" by Risto-Pekka Pölönen, Tampere University, Faculty of Medicine and Health Technology, Finland. The Heart Center, Tampere University Hospital, Tampere, Finland is affiliated to the ethical committee of Pirkanmaa Hospital District and performs research according to the guidelines of this regulatory body.

\section{Compliance with ethical standards}

Conflict of interest The authors declare that they have no conflict of interest. 
Ethical approval Approval from the Ethics Committee of Pirkanmaa Hospital District was given to conduct the research on hiPSC lines (Aalto-Setälä R08070). Patients donating skin biopsies were provided with an informed consent in the Heart Hospital, Tampere University Hospital.

Open Access This article is distributed under the terms of the Creative Commons Attribution 4.0 International License (http://creativeco mmons.org/licenses/by/4.0/), which permits unrestricted use, distribution, and reproduction in any medium, provided you give appropriate credit to the original author(s) and the source, provide a link to the Creative Commons license, and indicate if changes were made.

\section{References}

1. Ohnuki M, Takahashi K, Yamanaka S (2009) Generation and characterization of human induced pluripotent stem cells. Curr Protoc Stem Cell Biol. https://doi.org/10.1002/9780470151808. $\mathrm{sc0} 4 \mathrm{a} 02 \mathrm{~s} 9$

2. Swan H, Piippo K, Viitasalo M et al (1999) Arrhythmic disorder mapped to chromosome 1q42-q43 causes malignant polymorphic ventricular tachycardia in structurally normal hearts. J Am Coll Cardiol 34:2035-2042

3. Roston TM, Van Petegem F, Sanatani S (2017) Catecholaminergic polymorphic ventricular tachycardia: a model for genotypespecific therapy. Curr Opin Cardiol 32:78-85

4. Zhang J, Zhou Q, Smith CD et al (2015) Non- $\beta$-blocking R-carvedilol enantiomer suppresses $\mathrm{Ca}^{2+}$ waves and stress-induced ventricular tachyarrhythmia without lowering heart rate or blood pressure. Biochem J 470:233-242

5. Hilliard FA, Steele DS, Laver D et al (2010) Flecainide inhibits arrhythmogenic $\mathrm{Ca}^{2+}$ waves by open state block of ryanodine receptor $\mathrm{Ca}^{2+}$ release channels and reduction of $\mathrm{Ca}^{2+}$ spark mass. J Mol Cell Cardiol 48:293-301

6. Zhou Q, Xiao J, Jiang D et al (2011) Carvedilol and its new analogs suppress arrhythmogenic store overload-induced $\mathrm{Ca}^{2+}$ release. Nat Med 17:1003-1009

7. Watanabe H, Chopra N, Laver D et al (2009) Flecainide prevents catecholaminergic polymorphic ventricular tachycardia in mice and humans. Nat Med 15:380-383

8. Bers DM (2002) Cardiac excitation-contraction coupling. Nature 415:198-205

9. Grunnet M (2010) Repolarization of the cardiac action potential. Does an increase in repolarization capacity constitute a new antiarrhythmic principle? Acta Physiol (Oxford, England) 198(Suppl 676): $1-48$

10. Sato D, Bartos D, Ginsburg K, Bers D (2014) Depolarization of cardiac membrane potential synchronizes calcium sparks and waves in tissue. Biophys J 107:1313-1317

11. Cyganek L, Tiburcy M, Sekeres K et al (2018) Deep phenotyping of human induced pluripotent stem cell-derived atrial and ventricular cardiomyocytes. JCI Insight. https://doi.org/10.1172/jci. insight.99941

12. Huebsch N, Loskill P, Mandegar MA et al (2015) Automated video-based analysis of contractility and calcium flux in humaninduced pluripotent stem cell-derived cardiomyocytes cultured over different spatial scales. Tissue Eng C 21:467-479

13. Kijlstra JD, Hu D, Mittal N et al (2015) Integrated analysis of contractile kinetics, force generation, and electrical activity in single human stem cell-derived cardiomyocytes. Stem Cell Rep 5:1226-1238
14. Hansen KJ, Favreau JT, Gershlak JR, Laflamme MA, Albrecht DR, Gaudette GR (2017) Optical method to quantify mechanical contraction and calcium transients of human pluripotent stem cellderived cardiomyocytes. Tissue Eng C 23:445-454

15. Ahola A, Pölönen R, Aalto-Setälä K, Hyttinen J (2018) Simultaneous measurement of contraction and calcium transients in stem cell derived cardiomyocytes. Ann Biomed Eng 46:148-158

16. Spencer CI, Baba S, Nakamura K et al (2014) Calcium transients closely reflect prolonged action potentials in iPSC models of inherited cardiac arrhythmia. Stem Cell Rep 3:269-281

17. Lee P, Klos M, Bollensdorff C et al (2012) Simultaneous voltage and calcium mapping of genetically purified human induced pluripotent stem cell-derived cardiac myocyte monolayers. Circ Res 110:1556-1563

18. Prajapati C, Pölönen R, Aalto-Setälä K (2018) Simultaneous recordings of action potentials and calcium transients from human induced pluripotent stem cell derived cardiomyocytes. Biol Open. https://doi.org/10.1242/bio.035030

19. Dempsey GT, Chaudhary KW, Atwater N et al (2016) Cardiotoxicity screening with simultaneous optogenetic pacing, voltage imaging and calcium imaging. J Pharmacol Toxicol Methods 81:240-250

20. Penttinen K, Swan H, Vanninen S et al (2015) Antiarrhythmic effects of dantrolene in patients with catecholaminergic polymorphic ventricular tachycardia and replication of the responses using iPSC models. PLoS ONE 10:e125366

21. Paavola J, Väänänen H, Larsson K et al (2016) Slowed depolarization and irregular repolarization in catecholaminergic polymorphic ventricular tachycardia: a study from cellular $\mathrm{Ca}^{2+}$ transients and action potentials to clinical monophasic action potentials and electrocardiography. Europace 18:1599-1607

22. Pölönen RP, Penttinen K, Swan H, Aalto-Setälä K (2018) Antiarrhythmic effects of carvedilol and flecainide in cardiomyocytes derived from catecholaminergic polymorphic ventricular tachycardia patients. Stem Cells Int 2018:e9109503

23. Kujala K, Paavola J, Lahti A et al (2012) Cell model of catecholaminergic polymorphic ventricular tachycardia reveals early and delayed afterdepolarizations. PLoS ONE 7:e44660

24. Mummery C, Ward-van Oostwaard D, Doevendans P et al (2003) Differentiation of human embryonic stem cells to cardiomyocytes: role of coculture with visceral endoderm-like cells. Circulation 107:2733-2740

25. van Meer BJ, Sala L, Tertoolen LGJ, Smith GL, Burton FL, Mummery CL (2018) Quantification of muscle contraction in vitro and in vivo using MUSCLEMOTION Software: from stem cellderived cardiomyocytes to zebrafish and human hearts. Curr Protoc Hum Genet 99:e67

26. Priori SG, Napolitano C, Tiso N et al (2001) Mutations in the cardiac ryanodine receptor gene (hRyR2) underlie catecholaminergic polymorphic ventricular tachycardia. Circulation 103:196-200

27. Liu N, Colombi B, Memmi M et al (2006) Arrhythmogenesis in catecholaminergic polymorphic ventricular tachycardia: insights from a RyR2 R4496C knock-in mouse model. Circ Res 99:292-298

28. Cerrone M, Noujaim SF, Tolkacheva EG et al (2007) Arrhythmogenic mechanisms in a mouse model of catecholaminergic polymorphic ventricular tachycardia. Circ Res 101:1039-1048

29. Davis RP, van den Berg CW, Casini S, Braam SR, Mummery CL (2011) Pluripotent stem cell models of cardiac disease and their implication for drug discovery and development. Trends Mol Med 17:475-484

30. Fatima A, Xu G, Shao K et al (2011) In vitro modeling of ryanodine receptor 2 dysfunction using human induced pluripotent stem cells. Cell Physiol Biochem 28:579-592

31. Itzhaki I, Maizels L, Huber I et al (2012) Modeling of catecholaminergic polymorphic ventricular tachycardia with 
patient-specific human-induced pluripotent stem cells. J Am Coll Cardiol 60:990-1000

32. Novak A, Lorber A, Itskovitz-Eldor J, Binah O (2012) Modeling catecholaminergic polymorphic ventricular tachycardia using induced pluripotent stem cell-derived cardiomyocytes. Rambam Maimonides Med J 3:e0015

33. Novak A, Barad L, Zeevi-Levin N et al (2012) Cardiomyocytes generated from CPVTD307H patients are arrhythmogenic in response to $\beta$-adrenergic stimulation. J Cell Mol Med 16:468-482

34. Bhuiyan ZA, van den Berg MP, van Tintelen JP et al (2007) Expanding spectrum of human RYR2-related disease: new electrocardiographic, structural, and genetic features. Circulation 116:1569-1576

35. Lobo P, Kimlicka L, Tung C, Van Petegem F (2011) The deletion of exon 3 in the cardiac ryanodine receptor is rescued by $\beta$ strand switching. Structure 19:790-798

36. Marjamaa A, Laitinen-Forsblom P, Lahtinen AM et al (2009) Search for cardiac calcium cycling gene mutations in familial ventricular arrhythmias resembling catecholaminergic polymorphic ventricular tachycardia. BMC Med Genet 10:12

37. Peng W, Shen H, Wu J, Guo W, Pan X, Wang R, Chen SRW, Yan N (2016) Structural basis for the gating mechanism of the type 2 ryanodine receptor RyR2. Science 354(6310):aah5324

38. Tang Y, Tian X, Wang R, Fill M, Chen SRW (2012) Abnormal termination of Ca release is a common defect of RyR2 mutations associated with cardiomyopathies. Circ Res 110(7):968-977
39. Sato D, Shiferaw Y, Garfinkel A, Weiss JN, Qu Z, Karma A (2006) Spatially discordant alternans in cardiac tissue: role of calcium cycling. Circ Res 99:520-527

40. Xiao Z, Guo W, Sun B et al (2016) Enhanced cytosolic $\mathrm{Ca}^{2+}$ activation underlies a common defect of central domain cardiac ryanodine receptor mutations linked to arrhythmias. J Biol Chem 291:24528-24537

41. Paci M, Pölönen R, Cori D et al (2018) Automatic optimization of an in silico model of human iPSC derived cardiomyocytes recapitulating calcium handling abnormalities. Front Physiol 9:709

42. Kane C, Terracciano CMN (2017) Concise reviews: criteria for chamber-specific categorization of human cardiac myocytes derived from pluripotent stem cells. Stem Cells 8:1881-1897

43. Goversen B, van der Heyden, Marcel AG, van Veen TAB, de Boer TP (2018) The immature electrophysiological phenotype of iPSC-CMs still hampers in vitro drug screening: special focus on IK1. Pharmacol Ther 183:127-136

44. Lahti AL, Kujala VJ, Chapman H et al (2012) Model for long QT syndrome type 2 using human iPS cells demonstrates arrhythmogenic characteristics in cell culture. Dis Model Mech 5:220-230

Publisher's Note Springer Nature remains neutral with regard to jurisdictional claims in published maps and institutional affiliations. 